\title{
Meixner-Pollaczek polynomials and the Heisenberg algebra
}

\author{
Tom H. Koornwinder \\ Centre for Mathematics and Computer Science, P. O. Box 4079, 1009 AB Amsterdam, The Netherlands
}

(Received 12 July 1988; accepted for publication 9 November 1988)

\begin{abstract}
An alternative proof is given for the connection between a system of continuous Hahn polynomials and identities for symmetric elements in the Heisenberg algebra, which was first observed by Bender, Mead, and Pinsky [Phys. Rev. Lett. 56, 2445 (1986); J. Math. Phys. 28, 509 (1987) ]. The continuous Hahn polynomials turn out to be Meixner-Pollaczek polynomials. Use is made of the connection between Laguerre polynomials and MeixnerPollaczek polynomials, the Rodrigues formula for Laguerre polynomials, an operational formula involving Meixner-Pollaczek polynomials, and the Schrödinger model for the irreducible unitary representations of the three-dimensional Heisenberg group.
\end{abstract}

\section{INTRODUCTION}

In two recent papers ${ }^{1,2}$ Bender, Mead, and Pinsky discussed the connection between certain continuous Hahn polynomials and symmetrizations of elements in the Heisenberg algebra. They showed that, if

$$
[q, p]=i
$$

and $T_{m, n}$ is the sum of all possible terms containing $m$ factors of $p$ and $n$ factors of $q$, then

$$
T_{n, n}=\text { const } S_{n}\left(T_{1,1}\right) \text {, }
$$

for some polynomial $S_{n}$ of degree $n$, which turns out to be the orthogonal polynomial of degree $n$ on $\mathbb{R}$ with respect to the weight function $x \mapsto 1 / \operatorname{ch}(\pi x / 2)$. However, the actual proof of this result is not very clear from these two papers.

In the present paper we give an alternative proof of (1.1). First, in Sec. II, we observe a transformation connecting certain continuous Hahn polynomials, in particular, the above polynomials $S_{n}$ to certain Meixner-Pollaczek polynomials. Next, in Sec. III we use a Mellin transform relating Laguerre polynomials and Meixner-Pollaczek polynomials and the Rodrigues formula for Laguerre polynomials in order to derive an operational formula involving Meixner-Pollaczek polynomials. Finally, in Sec. IV we use this operational formula in order to derive formula (1.1). Here we make use of the Schrödinger model for the irreducible unitary representations of the Heisenberg group.

\section{ON CONTINUOUS HAHN POLYNOMIALS EXPRESSIBLE AS MEIXNER-POLLACZEK POLYNOMIALS}

Continuous Hahn polynomials are defined by

$$
\begin{aligned}
p_{n}(x ; a, b, c, d): & \\
= & i^{n} \frac{(a+c)_{n}(a+d)_{n}}{n} \\
& \times{ }_{3} F_{2}\left(\begin{array}{c}
-n, n+a+b+c+d-1, a+i x \\
a+c, a+d
\end{array} ; 1\right) .
\end{aligned}
$$

If $c=\bar{a}, d=\bar{b}$ and $\operatorname{Re} a, \operatorname{Re} b>0$, then they are orthogonal on $(-\infty, \infty)$ with respect to the weight function

$w(x):=\Gamma(a+i x) \Gamma(b+i x) \Gamma(c-i x) \Gamma(d-i x)$.
See Refs. 3 and 4, but read $a+i x$ instead of $a-i x$ in formula (3) of Ref. 4.

Meixner-Pollaczek polynomials are defined by

$$
P_{n}^{(a)}(x ; \phi):=e_{2}^{i n \phi}{ }_{2} F_{1}\left(-n, a+i x ; 2 a ; 1-e^{-2 i \phi}\right) .
$$

If $a>0$ and $0<\phi<\pi$, they are orthogonal on $(-\infty, \infty)$ with respect to the weight function

$$
w(x)=e^{(2 \phi-\pi) x}|\Gamma(a+i x)|^{2} .
$$

See Refs. 5 and 6 and, for standardized notation, the Appendix of Ref. 7.

For $a=c=b-\frac{1}{2}=d-\frac{1}{2}>0$ the weight function (2.2) becomes

$$
w(x)=2^{-4 a+2} \pi|\Gamma(2 a+2 i x)|^{2} .
$$

On comparing with (2.4) we conclude that

$$
p_{n}\left(x ; a, a+\frac{1}{2}, a, a+\frac{1}{2}\right)=\text { const } P_{n}^{(2 a)}\left(2 x ; \frac{1}{2} \pi\right) .
$$

The constant can be computed by comparing coefficients of $x^{n}$. We obtain

$$
\begin{aligned}
p_{n}\left(x ; a, a+\frac{1}{2}, a, a+\frac{1}{2}\right)= & {\left[(2 a)_{n}\left(2 a+\frac{1}{2}\right)_{n} / n !\right] } \\
& \times P_{n}^{(2 a)}\left(2 x ; \frac{1}{2} \pi\right) .
\end{aligned}
$$

In terms of hypergeometric functions this formula reads

$$
{ }_{3} F_{2}\left(\begin{array}{c}
-n, n+4 a, a+i x \\
2 a, 2 a+\frac{1}{2}
\end{array} \mid 1\right)={ }_{2} F_{1}(-n, 2 a+2 i x ; 4 a ; 2) .
$$

This identity can also be obtained from Ref. 8 ,

${ }_{4} F_{3}\left(\begin{array}{c}a, b, n+2 c,-n \\ a+b+\frac{1}{2}, c, c+\frac{1}{2}\end{array} ;\right)={ }_{3} F_{2}\left(\begin{array}{c}2 a, 2 b,-n \\ a+b+\frac{1}{2}, 2 c\end{array} ; 1\right)$,

by letting $b \rightarrow \infty$.

For $a:=\frac{1}{4}$ the weight function (2.5) becomes $w(x)=2 \pi^{2} / \operatorname{ch}(2 \pi x)$.

In particular, we find for the polynomials $S_{n}$ introduced in Sec. I, which were identified with special continuous Hahn polynomials in Ref. 2, that they can be written as MeixnerPollaczek polynomials:

$$
S_{n}(x)=\text { const } P_{n}^{(1 / 2)}\left(\frac{1}{2} x, \frac{1}{2} \pi\right) .
$$




\section{AN OPERATIONAL FORMULA INVOLVING MEIXNER-POLLACZEK POLYNOMIALS}

Recall that we can obtain the Mellin transform pair,

$$
\begin{aligned}
& G(\lambda)=\int_{0}^{\infty} F(\tau) \tau^{-1-i \lambda} d \tau, \\
& F(\tau)=(2 \pi)^{-1} \int_{-\infty}^{\infty} G(\lambda) \tau^{i \lambda} d \lambda,
\end{aligned}
$$

from the Fourier transform pair,

$$
\begin{aligned}
& g(\lambda)=\int_{-\infty}^{\infty} f(t) e^{-2 \pi i \lambda t} d t, \\
& f(t)=\int_{-\infty}^{\infty} g(\lambda) e^{2 \pi \lambda t} d \lambda,
\end{aligned}
$$

by making the substitutions

$$
\tau=e^{2 \pi t}, \quad F(\tau)=f(t), \quad G(\lambda)=2 \pi g(\lambda)
$$

in (3.2). In particular, Mellin inversion in (3.1) is valid if the function $t \mapsto F\left(e^{2 \pi t}\right)$ belongs to the class $\mathscr{S}$ of rapidly decreasing $C^{\infty}$ function on $\mathbb{R}$. If $F_{1}, F_{2}$ are two such functions and $G_{1}, G_{2}$ their Mellin transforms then we have the Parseval formula

$$
\int_{0}^{\infty} F_{1}(\tau) \overline{F_{2}(\tau)} \frac{d \tau}{\tau}=\int_{-\infty}^{\infty} G_{1}(\lambda) \overline{G_{2}(\lambda)} \frac{d \lambda}{2 \pi} .
$$

Proposition 3.1: For $a>0$ and $0<\phi<\pi$ Laguerre polynomials $x \mapsto L_{n}^{2 a-1}(x)$ and Meixner-Pollaczek polynomials $\lambda \mapsto P_{n}^{(a)}(\lambda ; \phi)$ are mapped onto each other by the Mellin transform in the following way:

$$
\begin{aligned}
\int_{0}^{\infty} \frac{n ! e^{-i n \phi}}{(2 a)_{n}} e^{-(1 / 2) x(1+i \cot \phi)} x^{a} L_{n}^{2 a-1}(x) x^{-1-i \lambda} d x \\
\quad=e^{(i a-\lambda)[\phi-(1 / 2) \pi]}(2 \sin \phi)^{a-i \lambda} \Gamma(a-i \lambda) P_{n}^{(a)}(\lambda ; \phi) .
\end{aligned}
$$

Proof: The left-hand side can be rewritten as

$$
\begin{aligned}
e^{-i n \phi} & \sum_{k=0}^{n} \frac{(-n)_{k}}{(2 a)_{k} k !} \int_{0}^{\infty} e^{-(1 / 2) x(1+i \cot \phi)} x^{k+a-i \lambda-1} d x \\
= & e^{-i n \phi} \sum_{k=0}^{n} \frac{(-n)_{k}}{(2 a)_{k} k !} \frac{\Gamma(a-i \lambda+k)}{\left(\frac{1}{2}+\frac{1}{2} i \cot \phi\right)^{a-i \lambda+k}} \\
= & e^{-i n \phi} \Gamma(a-i \lambda)\left(1-e^{2 i \phi}\right)^{a-i \lambda} \\
& \times_{2} F_{1}\left(-n, a-i \lambda ; 2 a ; 1-e^{2 i \phi}\right) \\
= & e^{i n \phi} \Gamma(a-i \lambda)\left(1-e^{2 i \phi}\right)^{a-i \lambda} \\
& \times{ }_{2} F_{1}\left(-n, a+i \lambda ; 2 a ; 1-e^{-2 i \phi}\right),
\end{aligned}
$$

which can be rewritten as the right-hand side of (3.4).

It is possible $e^{9,10}$ to give an interpretation of the above proposition in the context of matrix elements of discrete series representations of $\mathrm{SL}(2, \mathbb{R})$.

Corollary 3.2: For $a>0$ and $0<\phi<\pi$ Laguerre polynomials can be expressed by the differentiation formula

$$
\begin{aligned}
& \frac{n ! e^{-i n \phi}}{(2 a)_{n}} e^{(1 / 2) x(1+i \cot \phi)} x^{a} L_{n}^{2 a-1}(x) \\
& \quad=P_{n}^{(a)}\left(-i x \frac{d}{d x} ; \phi\right)\left(e^{-(1 / 2) x(1+i \cot \phi)} x^{a}\right)
\end{aligned}
$$

Proof: In the left-hand side of (3.4) Mellin transform is taken of a function that belongs to the class $\mathscr{S}$ as a function of $t$, where $x=e^{t}$. Hence we can apply Mellin inversion [cf. (3.1) ] and we can write the left-hand side of (3.5) as

$$
\begin{aligned}
(2 \pi)^{-1} & \int_{-\infty}^{\infty} e^{(i a-\lambda)[\phi-(1 / 2) \pi]}(2 \sin \phi)^{a-i \lambda} \\
& \times \Gamma(a-i \lambda) P_{n}^{(a)}(\lambda ; \phi) x^{i \lambda} d \lambda \\
= & P_{n}^{(a)}\left(-i x \frac{d}{d x} ; \phi\right) \\
& \times\left[e^{(i a-\lambda)[\phi-(1 / 2) \pi]}(2 \sin \phi)^{a-i \lambda}\right. \\
& \left.\times \Gamma(a-i \lambda) x^{i \lambda}\right],
\end{aligned}
$$

which equals the right-hand side of (3.5).

By substitution of the Rodrigues formula

$$
n ! e^{-x} x^{\alpha} L_{n}^{\alpha}(x)=\left(\frac{d}{d x}\right)^{n}\left(e^{-x} x^{n+\alpha}\right)
$$

into (3.5) we obtain

$$
\begin{aligned}
\left(\frac{d}{d x}\right)^{n} & \left(e^{-x} x^{n+2 a-1}\right) \\
= & (2 a)_{n} e^{i n \phi} e^{-(1 / 2) x(1-i \cot g \phi)} x^{a-1} \\
& \times P_{n}^{(a)}\left(-i x \frac{d}{d x}, \phi\right)\left[e^{-(1 / 2) x(1+i \cot \phi)} x^{a}\right] .
\end{aligned}
$$

In particular, for $\phi=\frac{1}{2} \pi$ and $a=\frac{1}{2}$ we obtain

$$
\begin{aligned}
& \left(i \frac{d}{d x}\right)^{n}\left(e^{-x} x^{n}\right) \\
& \quad=n ! e^{-(1 / 2) x} P_{n}^{(1 / 2)}\left(i x \frac{d}{d x}+\frac{1}{2} i, \frac{1}{2} \pi\right)\left[e^{-(1 / 2) x}\right] .
\end{aligned}
$$

Hence for arbitrary $v \in \mathbb{C}$,

$$
\begin{aligned}
& e^{i v x}\left(i \frac{d}{d x}\right)^{n}\left(x^{n} e^{-2 i v x}\right) \\
& \quad=n ! P_{n}^{(1 / 2)}\left(i x \frac{d}{d x}+\frac{1}{2} i, \frac{1}{2} \pi\right)\left[e^{-i v x}\right] .
\end{aligned}
$$

\section{PROOF OF THE BENDER-MEAD-PINSKY RESULT}

Consider the Heisenberg group $H_{1}$, which is $\mathbb{R}^{3}$ equipped with the multiplication rule

$$
\begin{aligned}
(\xi, \eta, \tau) & \left(\xi^{\prime}, \eta^{\prime}, \tau^{\prime}\right) \\
& =\left(\xi+\xi^{\prime}, \eta+\eta^{\prime}, \tau+\tau^{\prime}+\frac{1}{2}\left(\xi^{\prime} \eta-\xi \eta^{\prime}\right)\right) .
\end{aligned}
$$

Let $\lambda \in \mathbb{R} \backslash\{0\}$ and let $\pi_{\lambda}$ denote the unique (up to equivalence) irreducible unitary representation of $H_{1}$ such that

$$
\pi_{\lambda}(0,0, \tau)=e^{i \lambda \tau} I, \quad \tau \in \mathbb{R} .
$$

Then, with $\mu:=|\lambda|^{1 / 2}$ and $\epsilon:=\operatorname{sgn}(\lambda), \pi_{\lambda}$ can be realized on $L^{2}(\mathbb{R})$ by

$$
\begin{aligned}
& \left(\pi_{\lambda}(\xi, \eta, \tau) f\right)(x) \\
& \quad=e^{i \mu \xi x} e^{i \mu^{2}\left[\epsilon \tau+\left(\frac{1}{2}\right) \xi \eta\right]} f(x+\mu \eta), \quad f \in L^{2}(\mathbb{R}) .
\end{aligned}
$$

Let $X$ and $Y$ be the infinitesimal generators of the one-parameter subgroups of elements $(\xi, 0,0)$ and $(0, \eta, 0)$, respectively. Let $\sigma$ denote the symmetrization mapping ${ }^{11}$ from the symmetric algebra to the universal enveloping algebra of the Lie algebra of $H_{1}$, i.e., 


$$
\sigma\left(X_{1} \cdots X_{k}\right):=\frac{1}{k !} \sum_{s} X_{s(1)} \cdots X_{s(k)},
$$

where $s$ runs over all permutations of $\{1, \ldots, k\}$. Let $f$ be a $C^{\infty}$ function locally defined on $\mathbb{R}$. Then

$$
\begin{aligned}
& \left(\pi_{\lambda}(X) f\right)(x)=i \mu x f(x), \\
& \left(\pi_{\lambda}(Y) f\right)(x)=\mu f^{\prime}(x),
\end{aligned}
$$

and

$$
\begin{aligned}
& \left(\pi_{\lambda}\left(\sigma\left(X^{n} Y^{n}\right)\right) f\right)(x) \\
& \quad=\left.\left(\frac{\partial}{\partial \xi}\right)^{n}\left(\frac{\partial}{\partial \eta}\right)^{n}\left(e^{i \mu \xi x} e^{i \mu^{2}[\epsilon \tau+(1 / 2) \xi \eta]} f(x+\mu \eta)\right)\right|_{\xi, \eta, \tau=0} \\
& \quad=\left.\left(i \mu \frac{\partial}{\partial \eta}\right)^{n}\left(\left(x+\frac{1}{2} \mu \eta\right)^{n} f(x+\mu \eta)\right)\right|_{\eta=0} .
\end{aligned}
$$

Hence

$$
\begin{aligned}
& \left(\pi_{\lambda}\left(\sigma\left(X^{n} Y^{n}\right)\right) f\right)(x) \\
& =|\lambda|^{n}\left[i \frac{\partial}{\partial y}\right]^{n} \\
& \quad \times\left.\left(\left(x+\frac{1}{2} y\right)^{n} f(x+y)\right)\right|_{y=0} .
\end{aligned}
$$

For $n=1$ this simplifies to

$$
\left(\pi_{\lambda}(\sigma(X Y)) f\right)(x)=|\lambda|\left(i x \frac{\partial}{\partial x}+\frac{1}{2} i\right) f(x) .
$$

Let

$$
f_{v}(x):=e^{-i v x} \text {. }
$$

Then we obtain from (4.4), (3.7), and (4.5) that

$$
\begin{aligned}
\left(\pi_{\lambda}(\right. & \left.\left.\left(X^{n} Y^{n}\right)\right) f_{v}\right)(x) \\
& =\left.|\lambda|^{n}\left(i \frac{\partial}{\partial y}\right)^{n}\left(\left(x+\frac{1}{2} y\right)^{n} e^{-i v(x+y)}\right)\right|_{y=0} \\
& =2^{-n}|\lambda|^{n} e^{i \nu x}\left(i \frac{\partial}{\partial x}\right)^{n}\left(x^{n} e^{-2 i v x}\right) \\
& =2^{-n} n !|\lambda|^{n} P_{n}^{(1 / 2)}\left(i x \frac{d}{d x}+\frac{1}{2} i, \frac{1}{2} \pi\right)\left[e^{-i v x}\right] \\
& =2^{-n} n !|\lambda|^{n} P_{n}^{(1 / 2)}\left(|\lambda|^{-1} \pi_{\lambda}(\sigma(X Y)), \frac{1}{2} \pi\right)\left[f_{v}(x)\right] .
\end{aligned}
$$

Hence by integrating both sides against suitable functions of $v$, we obtain

$$
\pi_{\lambda}\left(\sigma\left(X^{n} Y^{n}\right)\right)=2^{-n} n !|\lambda|^{n} P_{n}^{(1 / 2)}\left(|\lambda|^{-1} \pi_{\lambda}(\sigma(X Y)), \frac{1}{2} \pi\right) .
$$

In view of (2.9) and (4.3) this becomes for $\lambda=1$ the result (1.1) of Ref. 1.

\section{ACKNOWLEDGMENT}

The author thanks G. Gasper for a reference to (2.8). 'C. M. Bender, L. R. Mead, and S. S. Pinsky, Phys. Rev. Lett. 56, 2445 (1986).

${ }^{2}$ C. M. Bender, L. R. Mead, and S. S. Pinsky, J. Math. Phys. 28, 509 (1987).

${ }^{3}$ N. M. Atakishiyev and S. K. Suslov, J. Phys. A 18, 1583 (1985).

${ }^{4}$ R. Askey, J. Phys. A 18, L1017 (1985).

${ }^{5}$ J. Meixner, J. London Math. Soc. 9, 6 (1934).

${ }^{6}$ F. Pollaczek, C. R. Acad. Sci. Paris 230, 1563 (1950).

${ }^{7}$ R. Askey and J. Wilson, Mem. Am. Math. Soc. 54, 319 (1985).

${ }^{8}$ W. N. Bailey, Proc. London Math. Soc. 29, 495 (1929).

${ }^{9}$ T. H. Koornwinder, "Group theoretic interpretations of Askey's scheme of hypergeometric orthogonal polynomials," "Orthogonal polynomials and their applications," Lecture Notes in Mathematics, Vol. 1329, edited by M. Alfaro et al. (Springer, Berlin, 1988), pp. 46-72.

${ }^{10}$ D. Basu and K. B. Wolf, J. Math. Phys. 23, 189 (1982).

${ }^{11} \mathrm{~S}$. Helgason, Groups and Geometric Analysis (Academic, New York, 1984). 